\title{
Acute visual loss and intraocular hemorrhages associated with endoscopic spinal surgery
}

\author{
Marilita M Moschos \\ Alexandros Rouvas \\ Alexios Papaspirou \\ Michael Apostolopoulos
}

Department of Ophthalmology, University of Athens, Greece
Correspondence: Marilita M Moschos 144, Kountouriotou street, I 8535 Piraeus, Greece

$\mathrm{Tel}+306944887319$

$\mathrm{Fax}+302104122139$

Email moschosmarilita@yahoo.fr
Purpose: To report a case of acute visual loss after endoscopic spinal surgery.

Methods: A patient was immediately referred to our department after epidural endoscopy with severe bilateral visual impairment and underwent complete ophthalmologic examination.

Results: Visual acuity was decreased in both eyes. Fundus examination revealed the presence of retinal and vitreous hemorrhages bilaterally. Four months later, visual acuity increased and the hemorrhages were remarkably resolved.

Conclusion: The present case represents an extremely rare incidence of intraocular hemorrhage following epiduroscopy.

Keywords: visual acuity, hemorrhage, intraocular, BCVA

\section{Case report}

A 45-year old man suffered from low back pain unresponsive to pharmacologic treatment, radiating to the left hip and left lower extremity, due to a back injury 4 years ago. Other medical history was noncontributory and he was otherwise healthy without hypertension, diabetes mellitus, cardiovascular disease, or blood clotting abnormalities.

The patient underwent an epidural endoscopy and endoscopic adhesiolysis in the level of $\mathrm{L}_{4} \mathrm{~L}_{5}$ and $\mathrm{O}_{5} \mathrm{~S}_{1}$ by infusion of $120 \mathrm{cc}$ of normal saline. At the end of the procedure the patient received an epidural injection of $80 \mathrm{mg}$ methylprednisolone.

After the operation the general condition of the patient was good without clinical signs of elevated cerebrospinal pressure and blood pressure and heart rate remained stable. However, a few hours later, he became aware of a major decrease of vision in both eyes and was immediately admitted to the department of ophthalmology.

At presentation, best corrected visual acuity (BCVA) was 20/400 RE and counting fingers LE. The ophthalmic examination revealed the presence of extensive scattered preretinal, subhyaloid, and subretinal hemorrhages in both eyes and especially in the LE (Figure 1). Ocular history before the operation did not reveal any ocular disease and BCVA was 20/20 in both eyes.

Three weeks after surgery, BCVA was 20/100 RE and counting fingers LE. Intraocular pressure and anterior segments showed no abnormalities in both eyes. Examination of the fundus showed mild vitreous hemorrhage with extensive intraretinal and subretinal hemorrhages involving the macula especially of the LE.

Four months after the operation, BCVA was 20/30 in both eyes. Despite the improved vision the patient still noted a central scotoma in both eyes. Fundus examination revealed a remarkable resolution of the retinal and subretinal hemorrhages bilaterally with mild pigmentary changes of the macula especially of the LE (Figure 2).

\section{Discussion}

Intraocular hemorrhages often occur in association with acute subarachnoid hemorrhage and secondary aneurysms of the anterior communicating and internal carotid arteries 


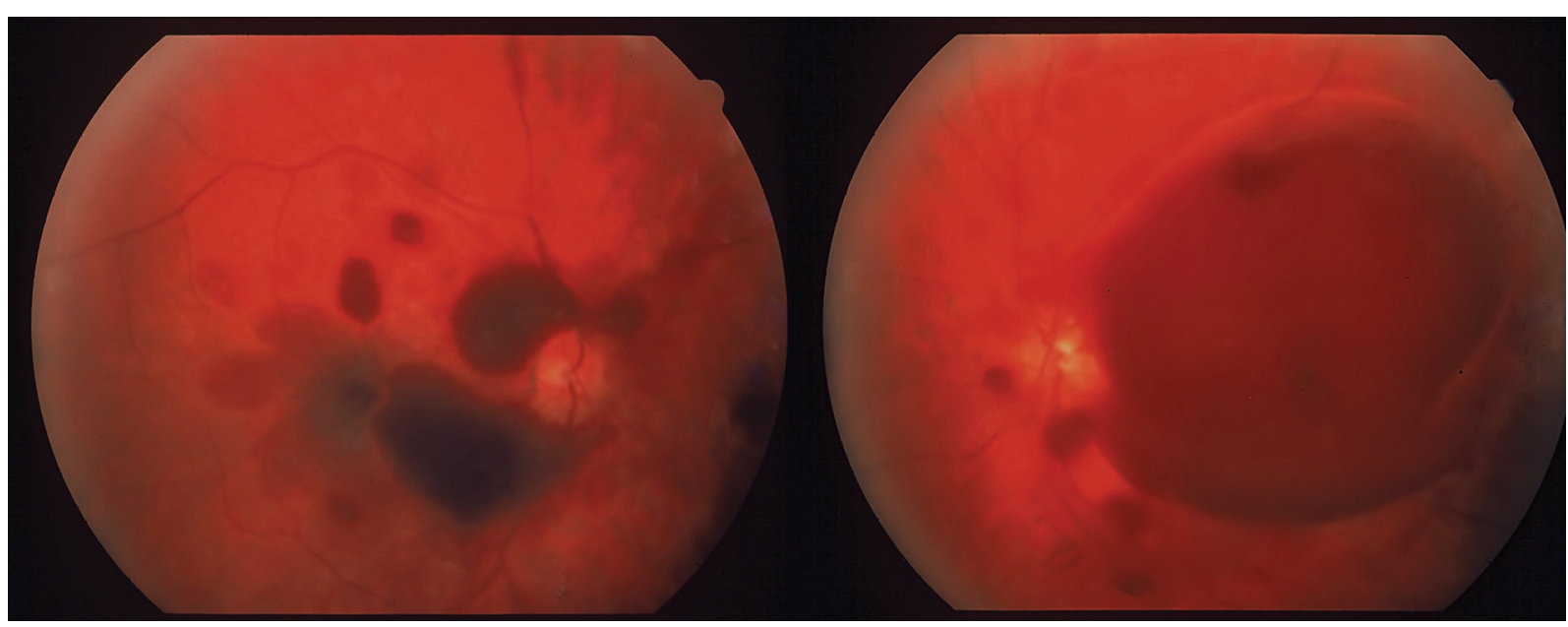

Figure I Fundus photograph of OD (left) and LE (right) showing subhyaloid, preretinal and retinal hemorrhages I week after the spinal surgery.

(Terson 1900). The present case represents an extremely rare incidence of intraocular hemorrhage following epiduroscopy and endoscopic adhesiolysis (Amirikia et al 2000; Brian et al 2005). Several mechanisms have been proposed to explain the occurrence of intraocular hemorrhage (Purdy et al 1998). It seems that a sudden increase of the epidural pressure after the epiduroscopy causes cerebrospinal fluid to effuse through the communication of the subarachnoid space within the optic nerve sheath and subsequently compresses the optic nerve and its vasculature. Specifically, the retinochoroidal anastomosis and the central retinal vein are occluded resulting in venous stasis allowing extravasation of blood through the vessels causing subhyaloid, retinal, and subretinal hemorrhages. The different types of hemorrhages suggest an acute generalized extravasation of blood within the various layers of the posterior segment opposed to the direct tracking of blood from within the optic nerve sheath. More particularly, intracranial pressure can be directly affected if the dura is punctured and fluid is added to the subarachnoid space. The visual prognosis is considered to be good, with recovery occurring within 6 months (Tabandeh 2000). Surgery may be required for persistent vitreous hemorrhage and secondary complications.

In conclusion the present case is rare and it represents a rare complication of a rather common operation. However, as endoscopic spinal procedures become widely established, it is anticipated that similar cases will be recognized more frequently in the future. Therefore, surgeons should be aware of the possibility of possible vision loss after this procedure.

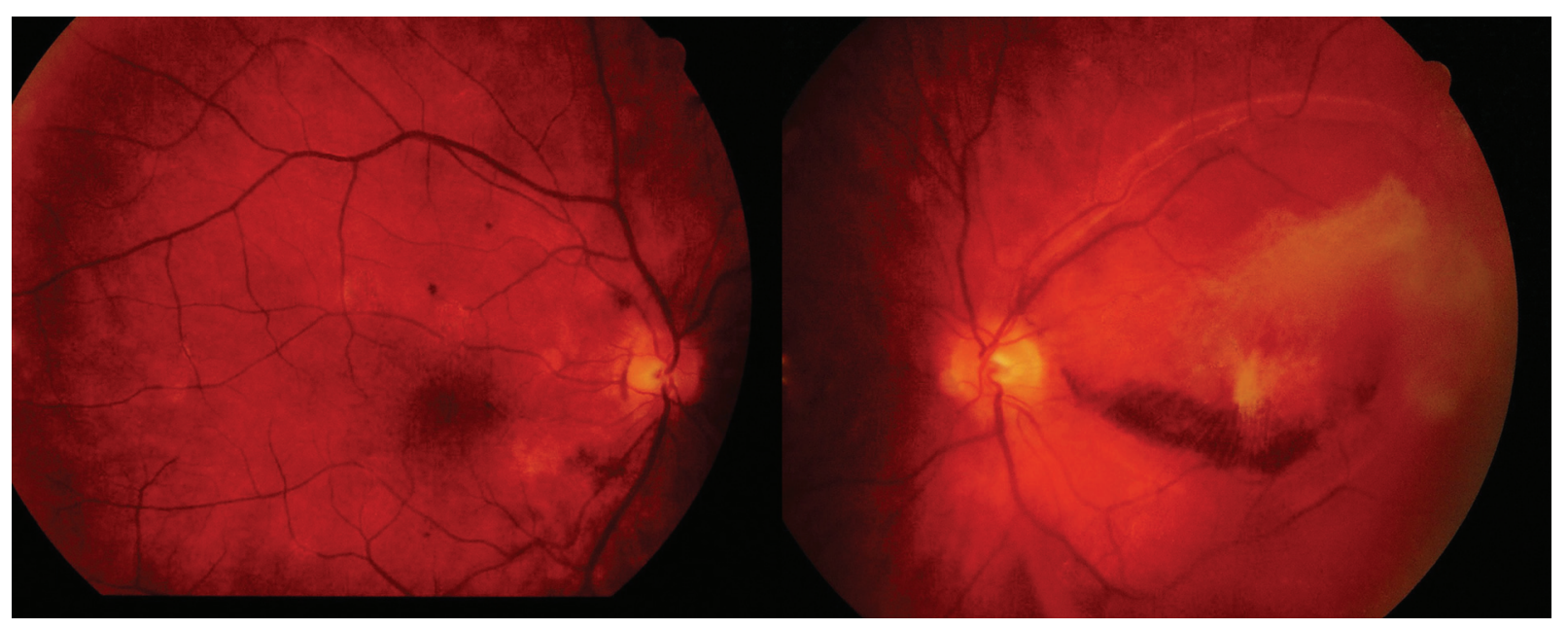

Figure 2 Fundus photograph of OD (left) and LE (right) showing the resolution of retinal and vitreous hemorrhages in both eyes. 


\section{Disclosure}

The authors report no conflicts of interest.

\section{References}

Amirikia A, Scott IU, Murray TG, et al. 2000. Acute bilateral visual loss associated with retinal hemorrhages following epiduroscopy. Arch Ophthalmol, 118:287-9.
Brian JG, Heavner EJ. 2005. Visual impairment following epidural fluid injections and epiduroscopy: A review. Pain Med, 6:367-74.

Purdy EP, Gurjit AS. 1998. Visual loss after lumbar epidural steroid injection. Anesth Analg, 86:119-22.

Tabandeh H. 2000. Intraocular hemorrhages associated with endoscopic spinal surgery. Am J Ophthalmol, 129:688-90.

Terson A. 1900. De 1' hémorragie dans cérébrale hémorragie dans le corps vitré au cours de 1' hémorragie cérébrale. Clin Ophtalmol, 6:309-12. 
